\title{
REAL ANALYTIC RADON TRANSFORMS ON RANK ONE SYMMETRIC SPACES
}

\author{
ERIC TODD QUINTO
}

(Communicated by J. Marshall Ash)

\begin{abstract}
Using microlocal techniques, we prove support theorems for Radon transforms with real analytic measures on horocycles in rank one symmetric spaces. We generalize Helgason's support theorem to this case and prove a new local support theorem.
\end{abstract}

\section{INTRODUCTION}

Radon transforms on symmetric spaces have a rich theory because of the extra structure that the groups provide. In this article, we will use this structure plus the theory of analytic Fourier integral operators to prove support theorems for real analytic Radon transforms on horocycles in symmetric spaces. This study is based on the pioneering work of Helgason (e.g., [He3, He4]) on group Radon transforms and Guillemin [G1, GS, G2] on Radon transforms as Fourier integral operators. Since then several authors have studied Radon transforms as Fourier integral operators [GU, BQ2]. The microlocal analysis is easiest for rank one, the case considered here.

Let $X$ be a real analytic manifold and let the manifold $Y$ parameterize certain subsets of $X$. Let $\mu$ be a weight function on each of these subsets. Let $R_{\mu}$ be a Radon transform that integrates functions on $X$ over the sets parameterized by $Y$ in the weight $\mu$. A typical support theorem for $R_{\mu}$ is as follows: given appropriate functions $f$ on $X$ and an appropriate subset $\mathscr{A}$ of $Y$, if $R f(\xi)=0$ for each submanifold $\xi$ in $\mathscr{A}$, then $f$ is zero on all points in the union of the submanifolds in $\mathscr{A}$. Cormack [Co], Solmon [So], and others have proven support theorems for transforms integrating over various curves and surfaces and with nonstandard measures (e.g., [Fi, O, Q2]). However, there are examples depending on the function class (e.g., [ShK]) or measure [Bo] for which support theorems do not hold. Support theorems are useful in partial differential equations [He4] and tomography [Q3].

The support theorems in this article are for the Radon transform on horocycles on rank one symmetric space with arbitrary nowhere zero real analytic

Received by the editors May 23, 1991.

1991 Mathematics Subject Classification. Primary 44A12; Secondary 58G15, 43A85.

Key words and phrases. Radon transform on horocycles, symmetric space, support theorems, microlocal analysis.

The author was partially supported by NSF grant MCS 8901203 . 
measures. Under the assumption that the measures are group invariant, Helgason [He4] proved Theorem 4.1 for symmetric spaces of all ranks using purely Lie algebraic techniques. Theorem 4.3 is new, even in the classical case. In our proofs, the theory of real analytic Fourier integral operators is used to deduce analytic smoothness of a distribution $f$ from support restrictions on $R_{\mu} f$ (Theorem 3.1, see also [G1]). Then a theorem of Hörmander Kawai and of Kashiwara [Hö, Ka] about analytic singularities and support is used to deduce support restrictions on $f$ from analytic smoothness of $f$. The Radon transform, $R_{\mu}$, and its dual, $R_{\mu}^{*}$, are defined using the group double fibration in $\S 2$; the microlocal analysis is given in $\S 3$; and the support theorems are proven in $\S 4$.

\section{THE POINT-HOROCYCLE DOUBLE FIBRATION}

Let $G$ be a real semisimple noncompact Lie group. Let $G=K A N$ be its Iwasawa decomposition and let $M$ be the centralizer of $A$ in $K$. The pointhorocycle double fibration is

$$
\begin{aligned}
Z= & G / M \stackrel{p_{Y}}{\longrightarrow} Y=G / M N \\
& \downarrow^{p_{X}} \\
X=G / K &
\end{aligned}
$$

where $p_{X}$ and $p_{Y}$ are the natural maps [He5].

The double fibration provides manifolds of integration for a Radon transform. For each $\xi \in Y$, there corresponds a unique closed horocycle in $X$,

$$
\hat{\xi}=p_{X} p_{Y}^{-1}(\{\xi\}) \quad \text { with measure } d m_{\xi}(x)
$$

and for each $x \in X$, there corresponds a unique compact subset of $Y$,

$$
\check{x}=p_{Y} p_{X}^{-1}(\{x\}) \quad \text { with measure } d m_{x}(\xi) \text {. }
$$

These measures are defined canonically in terms of the appropriate Haar measures on the groups. Because the maps in (2.1) are fibrations, all $\hat{\xi}$ are diffeomorphic (to $M N / M \simeq N$ ), and all $\check{x}$ are diffeomorphic (to $K / M$ ) $[\mathrm{He} 2$, He5]. The set $Z$ is called the incidence relation because $Z$ can be embedded in $X \times Y$ as $Z=\{(x, \xi) \in X \times Y \mid x \in \hat{\xi}\}$, and under this identification, the maps $p_{X}$ and $p_{Y}$ are projections.

These manifolds and measures define a Radon transform and its dual in the following way. Let $\mu(x, \xi)$ be a nowhere zero real analytic function for $(x, \xi) \in Z$. Let $f \in C_{c}(X)$, then the Radon transform of $f$ is defined by

$$
R_{\mu} f(\xi)=\int_{x \in \hat{\xi}} f(x) \mu(x, \xi) d m_{\xi}(x) .
$$

This is the integral of $f$ over the horocycle $\hat{\xi} \subset X$ with respect to the analytic measure $\mu d m_{\xi}$. The dual Radon transform of $g \in C(Y)$ is

$$
R_{\mu}^{*} g(x)=\int_{\xi \in \dot{x}} g(\xi) \mu(x, \xi) d m_{x}(\xi),
$$

the integral of $g$ over the compact set $\check{x} \subset Y$ of all horocycles through $x$ with respect to the analytic measure $\mu d m_{x}$. As in the classical case [He2] $R_{\mu}$ and 
$R_{\mu}^{*}$ are formally dual under the $G$ invariant measures on $X$ and $Y$. The Radon transform and its dual can be understood as push forwards of pull backs of measures on $X, Y$, and $Z$ [GS, p. 365].

We include the weight $\mu$ in the definition of the Radon transform (2.2) because canonical measures do not occur in general. Employing non-standard measures can help focus on properties intrinsic to the Radon transform rather than on specific symmetry relations that are valid only for special cases. Also, our proofs, involving microlocal analysis, do not require canonical measures.

\section{The RADON TRANSForm AS AN ANALytic FouriER INTEgRAL OPERATOR}

The key to the support theorems is the way that $R_{\mu}$ detects singularities, Theorem 3.1. Recall that $X$ is a real analytic manifold and so the analytic wave front set, $W F_{A}(f)$, of a distribution $f \in \mathscr{E}^{\prime}(X)$ is defined using local coordinates and the definition in $\mathbb{R}^{n}[\mathrm{Tr}]$.

Theorem 3.1. Let $G$ be a semisimple Lie group of real rank one. Let $R_{\mu}$ be a Radon transform on horocycles with a weight function $\mu$ that is real analytic and never zero. Let $f \in \mathscr{E}^{\prime}(X)$ and let $\xi_{o}$ be a given horocycle. Assume $R_{\mu} f=0$ for all horocycles in an open neighborhood of $\xi_{0}$. Let $N^{*} \hat{\xi}_{0}$ be the conormal bundle of $\hat{\xi}_{0}$ in $T^{*} X$. Then $W F_{A}(f) \cap N^{*} \hat{\xi}_{0}=\varnothing$.

The essential calculations in this proof were done by Victor Guillemin [G1] in unpublished work. Guillemin proves general conditions on an arbitrary group fibration for $R_{\mu}^{*} R_{\mu}$ to be an elliptic pseudodifferential operator. For rank greater than one, $R_{\mu}^{*} R_{\mu}$ is not elliptic and Theorem 3.1 is not true. We prove a weaker statement for rank greater than one in [GQ] (see end of $\S 3$ ).

Proof of Theorem 3.1. Because Guillemin's proof [G1] was never published, the relevant details are given here. The key to Theorem 3.1 is an understanding of the microlocal analysis of the operators $R_{\mu}$ and $R_{\mu}^{*}$. This is gotten from the diagram on the cotangent level corresponding to the double fibration (2.1):

$$
\begin{gathered}
\Gamma=N^{*} Z \backslash 0 \stackrel{\pi_{Y}}{\longrightarrow} T^{*}(Y) \backslash 0 \\
\downarrow \pi_{X} \\
T^{*}(X) \backslash 0
\end{gathered}
$$

where $\pi_{X}$ and $\pi_{Y}$ are the natural projections.

Without loss of generality, we can assume $\xi_{o}$ is the identity coset in $Y$. The set $p_{Y}^{-1}\left(\xi_{o}\right)=M N / M \simeq N$ is the orbit of $M N$ in $Z=G / M$ (see [He2, Theorem 3.4]). Let $\Gamma_{o}$ be the subset of $\Gamma$ lying above $p_{Y}^{-1}\left(\xi_{o}\right)$. We first show

Lemma 3.2. $\pi_{X}: \Gamma_{o} \rightarrow N^{*} \hat{\xi}_{0} \backslash 0$ is a diffeomorphism.

Proof. The argument is valid for any double fibration. First, note for $\left(x, \xi_{o}\right) \in Z$ that the sequence

$$
0 \longrightarrow T_{x} \hat{\xi}_{o} \stackrel{i}{\longrightarrow} T_{x} X \stackrel{j}{\longrightarrow} \frac{T_{\left(x, \xi_{o}\right)}(X \times Y)}{T_{\left(x, \xi_{o}\right)} Z} \longrightarrow 0
$$

is exact where $i$ is the inclusion and for $v \in T_{x} X, j(v)$ is the equivalence class of $(v, 0)$ in the quotient space in (3.2). Clearly im $i=\operatorname{ker} j$ as $v \in T_{x} \hat{\xi}_{o}$ iff 
$(v, 0) \in T_{\left(x, \xi_{o}\right)} Z$. The map $j$ is onto because $p_{Y}: Z \rightarrow Y$ is a submersion: let $(v, w) \in T_{\left(x, \xi_{o}\right)}(X \times Y)$, then there is a $\bar{v} \in T_{x} X$ such that $(\bar{v}, w) \in T_{\left(x, \xi_{o}\right)} Z$; thus $(v, w)$ is in the same equivalence class as $(v-\bar{v}, 0)$, and so $j$ is onto. Taking the dual diagram of (3.2) shows that $\pi_{X}$, the dual map to $j$ in (3.2), is a diffeomorphism on each fiber of $\Gamma_{o}$. Now, because $Z$ is embedded in $X \times Y, p_{X}: p_{Y}^{-1}\left(\xi_{o}\right) \rightarrow \hat{\xi}_{o}$ is a diffeomorphism on the base, so the lemma is proved.

Theorem 3.1 follows from the properties of the map $\pi_{Y}$ in (3.1). Lemma 3.4 will imply that $\pi_{Y}$ is an injective immersion if $G$ has real rank one. This is the Bolker Assumption [GS; see also Q1, p. 335], and it implies that $\Gamma$ is a local canonical graph. Because of the assumptions on the measure $\mu$, it also implies that $R_{\mu}$ is an elliptic Fourier integral operator associated with $\Gamma$ [GS, see also Q1, p. 337 above (15)]. Although this result was proven in the $C^{\infty}$ category, it is true in the real analytic category [Bj, SKK, Ka]. The calculus of such operators allows one to prove that, if $R_{\mu} f=0$ for horocycles near $\xi_{o}$, then $W F_{A} f \cap\left[\pi_{X} \circ \pi_{Y}^{-1} T_{\xi_{o}}^{*} Y \backslash 0\right]=\varnothing$. However, by the definition of $\Gamma_{o}$ and Lemma 3.2, $\pi_{X} \circ \pi_{Y}^{-1} T_{\xi_{o}}^{*} Y \backslash 0=N^{*} \hat{\xi}_{o} \backslash 0$. This proves Theorem 3.1 assuming the Bolker Assumption.

The composition rules of Fourier integral operators also imply that $R_{\mu}^{*} R_{\mu}$ is an analytic elliptic pseudodifferential operator [GS, see also Q1, Theorem 2.1].

A translation argument on $\Gamma$ above the fibers of $Z \rightarrow Y$ shows that the Bolker Assumption is equivalent to $\pi_{Y}: \Gamma_{o} \rightarrow T_{\xi_{o}}^{*} Y$ being an injective immersion. Let

$$
\bar{\rho}=\pi_{Y} \circ \pi_{X}^{-1}: N^{*} \hat{\xi}_{o} \backslash 0 \rightarrow T_{\xi_{o}}^{*} Y \backslash 0 .
$$

By Lemma 3.2, the map $\pi_{Y}: \Gamma_{o} \rightarrow T_{\xi_{o}}^{*} Y$ is an injective immersion iff the map $\bar{\rho}$ is. To finish the proof, we will prove that $\bar{\rho}$ is an injective immersion (Lemma 3.4).

To this end, we will first express $\bar{\rho}$ in terms of the Lie algebras (Lemma 3.3). Let $\mathfrak{g}, \mathfrak{k}, \mathfrak{a}, \mathfrak{m}$, and $\mathfrak{n}$ be the Lie algebras of $G, K, A, M$, and $N$, respectively. Let $\mathfrak{g}=\mathfrak{k} \oplus \mathfrak{p}$ be the Cartan decomposition of $\mathfrak{g}$, and let $B$ denote the Killing form. Recall that $\left.B\right|_{\mathfrak{k}}$ is negative definite; $\left.B\right|_{\mathfrak{p}}$ is positive definite; and that $\mathfrak{g}=\mathfrak{k} \oplus \mathfrak{p}$ is an orthogonal decomposition with respect to $B$ [Hel, Proposition III 7.4]. Identify $\mathfrak{g}^{*}$ with $\mathfrak{g}$ using $B$. If $\mathfrak{h} \subset \mathfrak{g}$, then let

$$
\mathfrak{h}^{\perp}=\{S \in \mathfrak{g} \mid B(S, \mathfrak{h})=0\} .
$$

Under the identification of $\mathfrak{g}$ with $\mathfrak{g}^{*}, \mathfrak{h}^{\perp}$ is the "conormal space" of $\mathfrak{h}$. Thus

$$
\mathfrak{k}^{\perp}=\mathfrak{p} \text { and }(\mathfrak{m}+\mathfrak{n})^{\perp}=\mathfrak{a}+\mathfrak{n} .
$$

The second equality in (3.4) is true for the following reasons. As $\mathfrak{k} \perp \mathfrak{p}, \mathfrak{m} \perp \mathfrak{a}$. Recall that $\mathfrak{n}$ is the direct sum of the positive root spaces [Wa, 7.3.5]; $(\mathfrak{m}+\mathfrak{a})$ is the "zero root space" [Wa, 7.3.4]; and two root spaces are orthogonal under the Killing form if the sum of their roots is not zero (see [Hel, III 4.2]). These facts imply $(\mathfrak{m}+\mathfrak{n}) \perp(\mathfrak{a}+\mathfrak{n})$, and now a dimension argument using [Wa, 7.3.5] completes the proof.

Lemma 3.3. Let $G=K A N$ be the Iwasawa decomposition of a semisimple Lie group of any real rank and let $M$ be the centralizer of $A$ in $K$. Let $\xi_{o}$ be 
the horocycle $M N$. Then the conormal space $N^{*} \hat{\xi}_{o} \backslash 0=\{(g K, \operatorname{Ad}(g) S) \mid g \in$ $M N, S \in \mathfrak{a} \backslash 0\}$ and the cotangent space $T_{\xi_{o}}^{*} Y=\mathfrak{a}+\mathfrak{n}$. The map $\bar{\rho}=\pi_{Y} \circ \pi_{X}^{-1}$ is projection on the second factor

$$
\begin{aligned}
\bar{\rho}: N^{*} \hat{\xi}_{o} \backslash 0 & \rightarrow T_{\xi_{o}}^{*} Y \backslash 0 \\
(g K, \operatorname{Ad}(g) S) & \rightarrow \operatorname{Ad}(g) S .
\end{aligned}
$$

Proof. $T_{\xi_{o}} Y$ is identified with $\mathfrak{g} /(\mathfrak{m}+\mathfrak{n})$ and so $T_{\xi_{o}}^{*} Y$ can be identified with $(\mathfrak{m}+\mathfrak{n})^{\perp}=\mathfrak{a}+\mathfrak{n}$ by $(3.4)$.

Let $g \in M N$. Then the isotropy group of $g K$ in $X$ is $g K^{-1}$. Using this, we see $T_{g K} X=\mathfrak{g} /(\operatorname{Ad}(g) \mathfrak{k})$. The manifold $\hat{\xi}_{o}$ is canonically diffeomorphic to $M N / M \simeq N$ and so

$$
T_{g K} \hat{\xi}_{o}=\frac{\mathfrak{m}+\mathfrak{n}}{\operatorname{Ad}(g) \mathfrak{m}}=\frac{\operatorname{Ad}(g)(\mathfrak{m}+\mathfrak{n})+\operatorname{Ad}(g) \mathfrak{k}}{\operatorname{Ad}(g) \mathfrak{k}} .
$$

Note in the last equality that $\operatorname{Ad}(g)(\mathfrak{m}+\mathfrak{n})=\mathfrak{m}+\mathfrak{n}$ because $g \in M N$. Using (3.4) one sees that the conormal space

$$
N_{g K}^{*} \hat{\xi}_{o}=\operatorname{Ad}(g)\left[(\mathfrak{m}+\mathfrak{n})^{\perp} \cap \mathfrak{k}^{\perp}\right]=\operatorname{Ad}(g)[(\mathfrak{a}+\mathfrak{n}) \cap \mathfrak{p}] .
$$

This simplification is valid because of the identification of $\mathfrak{g}$ with $\mathfrak{g}^{*}$ using the Killing form and that $B\left(\operatorname{Ad}\left(g^{-1}\right) S, T\right)=B(S, \operatorname{Ad}(g) T)$ for all $S \in \mathfrak{g}, T \in \mathfrak{g}$, and $g \in G$.

Let $\overline{\mathfrak{n}}$ be the sum of the negative root spaces. Now, because $\mathfrak{a} \subset \mathfrak{p}$ and $\mathfrak{n} \cap \overline{\mathfrak{n}}=\{0\}$, and because the Cartan involution maps $\mathfrak{p}$ onto itself and $\mathfrak{n}$ onto $\overline{\mathfrak{n}}$ [Wa, 7.3.5], $(\mathfrak{a}+\mathfrak{n}) \cap \mathfrak{p}=\mathfrak{a}$ so (3.6) becomes $N_{g K}^{*} \hat{\xi}_{o}=\operatorname{Ad}(g) \mathfrak{a}$. This proves that $N^{*} \hat{\xi}_{o}$ is as given in Lemma 3.3. Using this expression for $N^{*} \hat{\xi}_{o}$, Lemma 3.2 , and that the maps are all natural, one sees that $\bar{\rho}$ is just inclusion in the second factor.

The final step in the proof of Theorem 3.1 is the confirmation that the Bolker Assumption holds.

Lemma 3.4. If $G$ has real rank one, then the map $\bar{\rho}$ in (3.3) is an injective immersion.

Proof. Using Lemma 3.3, we first prove $\bar{\rho}$ is injective. Let $g \in M N$ be such that $\operatorname{Ad}(g) S \in \mathfrak{a}$ for some $S \in \mathfrak{a} \backslash 0$. As $\mathfrak{a}$ is one-dimensional, $g$ normalizes $\mathfrak{a}$. Let $g=m n$ for some $m \in M, n \in N$. Because $m$ centralizes $\mathfrak{a}$ and $g$ normalizes $\mathfrak{a}, n$ normalizes $\mathfrak{a}$. We now prove $n=e$. Assume $n \neq e$. Because $\operatorname{dim} \mathfrak{a}=1$, all restricted roots are 1-1 maps from $\mathfrak{a}$ to $\mathbb{R}$. Therefore the following map is 1-1 from $N$ to $\mathfrak{n}$ [He1, Lemma 4.6, p. 231]; $\phi\left(n_{1}\right)=$ $\operatorname{Ad}\left(n_{1}\right) S-S$. As $\phi(e)=0, \phi(n)=\operatorname{Ad}(n) S-S \in \mathfrak{n} \backslash 0$. Because $\mathfrak{n} \cap \mathfrak{a}=\{0\}$, $\operatorname{Ad}(n) S=S+\phi(n) \notin \mathfrak{a}$. Therefore, $n=e, g=m \in M$, and therefore $\bar{\rho}$ is injective.

To show $\bar{\rho}$ is an immersion at $\left(e M, S_{o}\right) \in N^{*} \hat{\xi}_{o} \backslash 0$, we construct an arbitrary path through $\left(e M, S_{o}\right)$ and take its derivative. Let $g(t)$ be a path in $M N$ with $g(0)=e$ and $\frac{d g}{d t}(0)=V_{o} \in \mathfrak{m}+\mathfrak{n}$, and let $S(t)$ be a path in $\mathfrak{a} \backslash 0$ with $S(0)=S_{o}$ and $\frac{d S}{d t}(0)=T_{o} \in \mathfrak{a}$. Then $v(t)=(g(t) K, \operatorname{Ad}(g(t)) S(t))$ is a path in $N^{*} \xi_{o} \backslash 0$ with derivative $\frac{d v}{d t}(0)=\left(V_{o}, \operatorname{ad}\left(V_{o}\right) S_{o}+T_{o}\right)$. Assume $d \bar{\rho} v(t)=\operatorname{ad}\left(V_{o}\right) S_{o}+T_{o}$ 
is zero. Therefore, $\operatorname{ad}\left(V_{o}\right) S_{o}=-T_{o} \in \mathfrak{a}$ and, since $\mathfrak{a}$ is one-dimensional and $S_{o} \neq 0, V_{o}$ normalizes a. Because $G$ has a finite Weyl group [Wa, 7.5.6], the normalizer of $\mathfrak{a}$ in $\mathfrak{k}$ is $\mathfrak{m}$. Because $\mathfrak{n}$ is the sum of the positive root spaces $[\mathrm{He} 1],[\mathfrak{a}, \mathfrak{n}] \subset \mathfrak{n}$. This shows that the normalizer of $\mathfrak{a}$ in $\mathfrak{g}$ is $\mathfrak{m}+\mathfrak{a}$ and so the normalizer of $\mathfrak{a}$ in $\mathfrak{m}+\mathfrak{n}$ is $\mathfrak{m}$. This shows $V_{0} \in \mathfrak{m}$ and so $\frac{d v}{d t}(0)=0$ and so $\bar{\rho}$ is an immersion.

When the real rank of $G$ is greater than one, the Bolker Assumption does not hold. [GQ] has a more restrictive theorem in this case under the other hypotheses of Theorem 3.1:

$$
W F_{A}(f) \cap N^{o}=\varnothing
$$

where $N^{o}=\{(g K, \operatorname{Ad}(g) S) \mid g \in M N, S \in \mathfrak{a} \backslash$ is regular $\} . S \in \mathfrak{a} \backslash 0$ is regular [He1] if $\alpha(S) \neq 0$ for all restricted roots $\alpha .^{1}$

\section{THE SUPPORT THEOREMS}

Theorem 4.1 is the generalization of Helgason's support theorem to noninvariant measures.

Theorem 4.1. Let $G$ be a semisimple Lie group of real rank one. Let $R_{\mu}$ be a Radon transform on horocycles with a weight function $\mu$ that is real analytic and never zero (2.1)-(2.2). Let $f \in \mathscr{E}^{\prime}(X)$ and let $\mathscr{A} \subset Y$ be an open connected subset. Assume $R_{\mu} f(\xi)=0$ for all horocycles $\xi \in \mathscr{A}$ and assume some horocycle in $\mathscr{A}$ is disjoint from $\operatorname{supp} f$. Then $\bigcup\{\hat{\xi} \mid \xi \in \mathscr{A}\}$ is disjoint from $\operatorname{supp} f$.

Theorem 4.1 implies a limited angle theorem; namely, let $V$ be an open neighborhood of $k_{0} \in K$. If $R_{\mu} f(\xi)=0$ for all horocycles kaMN with a $\in A$ and $k \in V$, then $f \equiv 0$. The proof uses the decomposition of $Y \cong K / M \times$ $A$ [He2, Proposition 3.6] and that $\mathscr{A}=\{k a M N \mid k \in V, a \in A\}$ is open unbounded and connected in $Y$.

Proof. The arguments are as in [BQ1] for the hyperplane transform. The first key idea is Theorem 3.1. Let $\xi_{1}$ be a horocycle in $\mathscr{A}$ that is disjoint from supp $f$ and let $\xi_{2}$ be an arbitrary horocycle in $\mathscr{A}$. Assume supp $f$ meets $\xi_{2}$. Let $\mathscr{P}$ be a path in $\mathscr{A}$ between $\xi_{1}$ and $\xi_{2}$ and let $\xi_{o}$ be the first horocycle in the path that meets supp $f$. As $R_{\mu} f=0$ for horocycles near $\xi_{o}$, the conclusion of Theorem 3.1 implies $W F_{A} f \cap N^{*} \hat{\xi}_{o}=\varnothing$. Furthermore, $f$ is zero on one side of $\hat{\xi}_{o}$.

The second key idea is a theorem of Kawai, Kashiwara, and Hörmander [Hö, Theorem 8.5.6]:

Lemma 4.2. Let $h \in \mathscr{E}^{\prime}(X)$ and assume $h$ is zero on one side of the horocycle $\hat{\xi}_{o}$. Let $x_{o} \in \hat{\xi}_{o} \cap \operatorname{supp} f$ and let $\left(x_{o}, \omega_{o}\right) \in N^{*} \hat{\xi}_{o} \backslash 0$. Then $\left(x_{o}, \omega_{o}\right) \in W F_{A} f$.

Hörmander's result is stated for $\mathbb{R}^{\mathrm{n}}$, but it can be proved in $X$ using real analytic local coordinates and the fact that in rank one spaces, horocycles are complete codimension one surfaces.

\footnotetext{
${ }^{1}$ If a has dimension one, then all restricted roots are one-to-one maps and so all $S \in \mathfrak{a} \backslash 0$ are regular.
} 
Applying Lemma 4.2 to $x_{0} \in \hat{\xi}_{0} \cap \operatorname{supp} f$ gives a contradiction that proves the theorem.

A local support theorem similar Globevnik's theorem [Gl] for the X-ray transform in $\mathbb{R}^{\mathrm{n}}$ also follows from Theorem 3.1 and Lemma 4.2.

Theorem 4.3. Let $G$ be a semisimple Lie group of real rank one. Let $R_{\mu}$ be a Radon transform on horocycles with a weight function $\mu$ that is real analytic and never zero (2.1)-(2.2). Let $B(r)$ be the open geodesic ball of radius $r$ in $X$. Let $f \in \mathscr{E}^{\prime}(X)$ and let $A_{1}=B(1) \cap \operatorname{supp} f$. Assume $A_{1}$ is compact, and assume $R_{\mu} f(\xi)=0$ for all horocycles $\hat{\xi}$ that meet $B(1)$ but not $B(1 / 2)$. Then $\operatorname{supp} f \cap[B(1) \backslash \mathrm{Cl} B(1 / 2)]=\varnothing$.

The hypotheses of Theorem 4.3 allow supp $f$ to include points outside $B(1)$. The choice of $B(1)$ and $B(1 / 2)$ are somewhat arbitrary and local support theorems can be proved for other sets determined by the horocycles disjoint from them.

Proof. Let $r$ be the smallest number such that $A_{1} \subset \mathrm{Cl} B(r)$, and assume the conclusion of Theorem 4.3 is false. As $A_{1}$ is a compact subset of $B(1), r \in$ $(1 / 2,1)$. Under these hypotheses, Theorem 3.1 implies $W F_{A} f \cap N^{*} \partial B(r)=\varnothing$ because through each $x \in \partial B(r)$ there is a horocycle lying outside $B(1 / 2)$ in each normal direction to $\partial B(r)$ [He2]. Applying Lemma 4.2 to $f_{1}=f \chi_{A_{1}}$ gives the contradiction that proves the theorem.

If the real rank of $G$ is greater than one, then (3.7) shows that some wavefront directions conormal to $\hat{\xi}_{o}$ are not detected by $R_{\mu}$. This is analogous to the X-ray transform on line complexes in $\mathbb{R}^{3}$ [BQ2] and on symmetric spaces [GU] where some wavefront directions conormal to lines in $Y$ are not detected by $R_{\mu}$. More subtle geometric arguments are needed to prove support theorems in these cases [BQ2, GQ].

\section{ACKNOWLEDGMENT}

The author thanks Fulton Gonzalez for many enlightening conversations about the geometry and algebra of symmetric spaces and Victor Guillemin for his inspiration.

\section{REFERENCES}

[Bo] J. Boman, An example of non-uniqueness for a generalized Radon transform, J. Analyse Math. (to appear).

[BQ1] J. Boman and E. T. Quinto, Support theorems for real analytic Radon transforms, Duke Math. J. 55 (1987), 943-948.

[BQ2] - Support theorems for real analytic Radon transforms on line complexes in $\mathbb{R}^{3}$, Trans. Amer. Math. Soc. (to appear).

[Co] A. Cormack, A Radon transform on a family or curves in the plane. I, Proc. Amer. Math. Soc. 83 (1981), 325-330; II, Proc. Amer. Math. Soc. 86 (1982), 293-298.

[Fi] D. Finch, Uniqueness of the attenuated X-ray transform in the physical range, Inverse Problems 2 (1986), 197-203.

[Gl] J. Globevnik, A support theorem for the X-ray transform, J. Math. Anal. Appl. (to appear).

[GQ] F. Gonzalez and E. T. Quinto, Microlocal analysis and Radon transforms on higher rank symmetric spaces, 1992. 
[GU] A. Greenleaf and G. Uhlmann, Non-local inversion formulas in integral geometry, Duke J. Math. 58 (1989), 205-240.

[G1] V. Guillemin, Some remarks on integral geometry, unpublished, 1975.

[G2] _ On some results of Gelfand in integral geometry, Proc. Sympos. Pure Math., vol. 43, Amer. Math. Soc., Providence, RI, 1985, pp. 149-155.

[GS] V. Guillemin and S. Sternberg, Geometric asymptotics, Amer. Math. Soc., Providence, RI, 1977.

[He1] S. Helgason, Differential geometry and symmetric spaces, Academic Press, New York, 1962.

[He2] _ Duality and Radon transform for symmetric spaces, Amer J. Math. 85 (1963), 667-692.

[He3] _ A duality for symmetric spaces, with applications to group representations, Adv. in Math. 5 (1970), 153-180.

[He4] _ The surjectivity of invariant differential operators on symmetric spaces. I, Ann. of Math. (2) 98 (1973), 451-479.

[He5] _ Groups and geometric analysis, Academic Press, New York, 1984.

[Hö] L. Hörmander, The analysis of linear partial differential operators. I, Springer, New York, 1983.

[Ka] A. Kaneko, Introduction to hyperfunctions, Kluwer, New York, 1989.

[O] J. Orloff, Invariant Radon transforms on a symmetric space, Contemp. Math., vol. 113, Amer. Math. Soc., Providence, RI, 1990, pp. 233-242.

[Q1] E.T. Quinto, The dependence of the generalized Radon transform on defining measures, Trans. Amer. Math. Soc. 257 (1980), 331-346.

[Q2] _ The invertibility of rotation invariant Radon transforms, J. Math. Anal. Appl. 91 (1983), 510-522; Erratum, J. Math. Anal. Appl. 94 (1983), 602-603.

[Q3] Tomographic reconstructions from incomplete data-numerical inversion of the exterior Radon transform, Inverse Problems 4 (1988), 867-876.

[SKK] M. Sato, T. Kawai, and M. Kashiwara, Hyperfunctions and pseudodifferential equations, Lecture Notes in Math., vol. 287, Springer-Verlag, New York, 1973, pp. 265-529.

[ShK] L. Shepp and J. Kruskal, Computerized tomography: the new medical X-ray technology, Amer. Math. Monthly 85 (1978), 420-439.

[So] D. Solmon, The X-ray transform, J. Math. Anal. Appl. 56 (1976), 51-66.

[Tr] F. Treves, Introduction to pseudodifferential and Fourier integral operators. I, Plenum Press, New York, 1980.

[Wa] N. Wallach, Harmonic analysis on homogeneous spaces, Dekker, New York, 1973.

Department of Mathematics, Tufts University, Medford, Massachusetts 02155

E-mail address: equinto@math.tufts.edu 